\title{
LOS DIFERENTES DATUM Y PROYECCIONES CARTOGRÁFICAS DE COSTA RICA: GENERALIDADES Y RELACIONES
}

\author{
DIFFERENT DATUM AND CARTOGRAPHIC \\ PROJECTIONS FROM COSTA RICA: GENERALITIES \\ AND RELATIONS
}

\author{
Jorge Moya Zamora ${ }^{1}$ \\ Bepsy Cedeño Montoya ${ }^{2}$ \\ Universidad Nacional de Costa Rica
}

http://dx.doi.org/10.15359/rgac.3-59.2

\begin{abstract}
RESUMEN
En Costa Rica se han implementado y usado una serie de proyecciones cartográficas desde la segunda mitad del siglo XX. La primera cartografía oficial del país, que funcionó durante varias décadas, estuvo basada en una proyección conforme de Lambert con dos paralelos de contacto. Con base en este sistemas cartográficos se definieron las denominadas las denominadas zonas norte y sur. Esta cartografía nacional consideró como superficie de referencia el elipsoide Clarke 1866, y como marco de referencia las coordenadas de los puntos provenientes de las cadenas de triangulación. A inicios de la década de 1990, se desarrolló un proyecto que tuvo dentro de sus objetivos primordiales dotar al país de una nueva red geodésica, basada en el posicionamiento satelital por medio de GPS. Las coordenadas ajustadas de los vértices de esta red referidas al elipsoide WGS84 constituyeron la base del llamado datum CR90, cuyos datos se usaron en la generación de

1 Dr.-Ingeniero, Centro Nacional de Procesamiento de Datos GNSS (CNPDG), Escuela de Topografía, Catastro y Geodesia. Universidad Nacional de Costa Rica. Correo Electrónico: jorge.moya.zamora@una.cr

2 Máster, Escuela de Ciencias Geográficas. Universidad Nacional de Costa Rica. Correo electrónico: bepsy. cedeno.montoya@una.cr
\end{abstract}

Fecha de recepción: 31 de agosto de 2016

Fecha de aceptación: 30 de enero de 2017 
Jorge Moya Zamora, Bepsy Cedeño Montoya. Different datum and cartographic projections from Costa Rica: generalities and relations

cartografía en proyección CRTM90. A finales de esa década, un proyecto de investigación desarrollado en la Escuela de Topografía, Catastro y Geodesia (ETCG) reunió las mediciones GPS de varios proyectos nacionales y realizó el primer ajuste de una red geodésica costarricense dentro del Marco Internacional Terrestre de Referencia (ITRF) del año 1994, dado a la época 1998,35 (Dörries y Roldán, 1998) y (Dörries y Roldán, 2004). Este nuevo datum se denominó como CR98, al cual se asoció la proyección CRTM98. A mediados de la década de los años 2000, se realizó la medición de otra red geodésica nacional dentro del ITRF2000, la cual constituye la base del datum oficial de Costa Rica, denominado como CR05 y la correspondiente cartografía oficial en proyección CRTM05. Las diferentes redes geodésicas que se han medido en Costa Rica y sus correspondientes proyecciones cartográficas implican que se cuente con una gran cantidad de información geoespacial, de la cual es fundamental conocer sus metadatos en aras de poder proyectarla al plano correcto o poder transformarla de manera adecuada. Si bien es cierto, para muchas de las aplicaciones prácticas la escala de trabajo no permite visualizar un efecto directo en el producto cartográfico, es indispensable el manejo adecuado de la información.

Palabras clave: datum Ocotepeque, datum CR90, datum CR98, datum CR05, proyecciones de Costa Rica.

\begin{abstract}
As of the second half of the 20th century, Costa Rica has been implemented and used a series of map projections. The first official map of the country that worked for decades was based on a projection of Lambert, with two contact parallels and defining the so-called northern and southern areas. This national mapping considered -as a reference surface, Clarke ellipsoid 1866, and the coordinates of the points from triangulation chains as a reference framework. In the early 1990s, a project whose main purpose was to provide the country with a new geodetic network based on satellite positioning via GPS was developed. The adjusted coordinates of the vertexes of this network on the WGS84 ellipsoid constituted the basis of datum CR90, whose data were used in generating CRTM90 projection mapping. At the end of that decade, a research project made by Escuela de Topografía, Catastro y Geodesia (ETCG), brought GPS measurements of several national projects, and made the first adjustment of Costa Rican geodetic network within the International Terrestrial Reference Framework (ITRF) 1994 (Dörries and Roldan, 1999) and (Dörries y Roldán, 2004). This new datum was designated as CR98, and it was related to the CRTM98 projection. In the mid-2000s, the measurement of another national geodetic network within ITRF2000 was performed, which constitutes the basis of the official datum of Costa Rica -known as CR05, and the corresponding official projection mapping CRTM05. The different geodetic networks that have been measured in Costa Rica and their corresponding map projections imply having a large amount of geospatial information, from which it is essential to know its metadata in order to be able to project it to the correct plane or to properly transform it. Although it is true that for many practical applications the working scale does not allow displaying a direct effect on the cartographic product, the proper management of information is essential.
\end{abstract}

Keywords: Ocotepeque datum, CR90 datum, CR98 datum, CR05 datum, projections of Costa Rica 


\section{Introducción}

Los diferentes datum de Costa Rica y sus proyecciones asociadas han generado una enorme cantidad de información geoespacial. El conocimiento general de cada uno de los datum, así como de la proyección cartográfica respectiva son indispensables para poder efectuar conversiones y transformaciones de coordenadas de una manera adecuada, pero sobre todo teniendo en cuenta el origen estas coordenadas. Actualmente, es de acatamiento obligatorio la georreferenciación de toda la información geoespacial al datum CR05 y su correspondiente proyección CR05, sin embargo, es evidente que se tiene mucha información referida a los antiguos datum y proyecciones, por lo que conocer adecuadamente los procesos de transformación, con el fin de poder vincular correctamente al datum oficial, es una obligación técnica y legal.

\section{Datum y proyecciones cartográficas de Costa Rica}

Costa Rica ha contado desde mediados de la segunda mitad del siglo XX hasta mediados a de la década del 2000 con diferentes sistemas cartográficos, basados fundamentalmente en las proyecciones de Lambert y variantes de la proyección Gauss-Krüger. A continuación, se hace una descripción general de estas proyecciones, estableciendo principalmente las diferencias en cada una, las cuales repercuten directamente con los datos generados en una o en otra. Los distintos acrónimos y siglas presentados en este trabajo son usados solamente para identificar y simplificar los nombres de los diferentes datum y proyecciones cartográficas, los mismos no son oficiales y se establecen solo como una guía para los lectores (ver lista de siglas y acrónimos).

\section{Datum convencional Ocotepeque y proyección Lambert}

Los vértices de la red geodésica clásica de Costa Rica provenientes de las antiguas cadenas de triangulación de los sectores Pacífico, Caribe, la cadena central y el sector norte constituyeron la base de la cartografía oficial del país durante más de 40 años. Las mediciones geodésicas de triangulación y las de algunas bases fueron realizadas con colaboración del antiguo Inter-American Geodetic Survey (IAGS) de los EE.UU (IAGS, 1950). Estos datos, posteriormente fueron procesados usando como punto fundamental el vértice Ocotepeque, ubicado en Honduras y usando como 
superficie de referencia el elipsoide Clarke 1866. Basados en estos puntos, se gestó el mapa básico de Costa Rica a escala 1:50000 con un total de 133 hojas con una cobertura individual de $10^{\prime} \mathrm{X} 15^{\prime}$, así como su generalización a escala 1:10000 para las 76 que cubren el Gran Área Metropolitana (GAM) y las 9 hojas a escala 1:200000.

La proyección Lambert implementada en Costa Rica se definió con dos paralelos de contacto, y generando dos sistemas de coordenadas cartográficas, denominados como Lambert Costa Rica Norte (LCRN) y Lambert Costa Rica Sur (LCRS) (IAGS, 1950). En el cuadro 1 se presentan los parámetros de los sistemas LCRN y LCRS, considerando para ambos el elipsoide Clarke 1866 con un valor para el semieje a de 6378206,4 m, para el semieje $b$ un valor de $6356583,8 \mathrm{~m}$ y para el cuadrado de la primera excentricidad un valor $\mathrm{e}^{2}=0,0067686579$ (IAGS, 1950).

Cuadro 1. Parámetros de los sistemas cartográficos Lambert Costa Rica

Norte y Lambert Costa Rica Sur

\begin{tabular}{|l|l|l|}
\hline \multicolumn{1}{|c|}{ Parámetro } & $\begin{array}{c}\text { Lambert Costa Rica } \\
\text { Norte (LCRN) }\end{array}$ & $\begin{array}{c}\text { Lambert Costa Rica } \\
\text { Sur (LCRS) }\end{array}$ \\
\hline Latitud del origen & $\mathrm{j}_{0}=10^{\circ} 28^{\prime} \mathrm{N}$ & $\mathrm{j}_{0}=09^{\circ} 00^{\prime} \mathrm{N}$ \\
\hline Longitud del origen & $\mathrm{l}_{0}=84^{\circ} 20^{\prime} \mathrm{O}$ & $\mathrm{l}_{0}=83^{\circ} 40^{\prime} \mathrm{O}$ \\
\hline Factor de escala en el origen & $\mathrm{k}_{0}=0,99995696$ & $\mathrm{k}_{0}=0,99995696$ \\
\hline Falso Norte & $\mathrm{FN}=271820,522 \mathrm{~m}$ & $\mathrm{FN}=327987,436 \mathrm{~m}$ \\
\hline Falso Este & $\mathrm{FE}=500000,000 \mathrm{~m}$ & $\mathrm{FE}=500000,000 \mathrm{~m}$ \\
\hline Radio desarrollado del paralelo & $\mathrm{R}_{0}=34528179,478 \mathrm{~m}$ & $\mathrm{R}_{0}=40272012,564 \mathrm{~m}$ \\
\hline Latitud de los paralelos de contacto & $\mathrm{j}_{1}=09^{\circ} 56^{\prime} \mathrm{N}$ & $\mathrm{j}_{1}=08^{\circ} 28^{\prime} \mathrm{N}$ \\
$\mathrm{j}_{2}=09^{\circ} 32^{\prime} \mathrm{N}$ \\
\hline
\end{tabular}

Fuente: Elaboración propia a partir de IAGS, 1950

El conjunto de ecuaciones (1), permite el cálculo de las coordenadas cartográficas (N, E) en los sistemas LCRN y LCRS a partir de las coordenadas geodésicas $(\varphi, \lambda)$. El cálculo $D \varphi$ debe realizarse en minutos de arco. Los coeficientes $\mathrm{a}_{1}, \ldots, \mathrm{a}_{6}$, dependen del elipsoide y para estas dos proyecciones sus valores están en el cuadro 2 . 


$$
\begin{aligned}
& \Delta \lambda=\lambda-\lambda_{0} \\
& \Delta \varphi=\varphi-\varphi_{0} \\
& \theta=\Delta \lambda \text { seno } \varphi_{0} \\
& \mathrm{R}=\mathrm{R}_{0}+\mathrm{a}_{1} \Delta \varphi+\mathrm{a}_{2} \Delta \varphi^{2}+\mathrm{a}_{3} \Delta \varphi^{3}+\mathrm{a}_{4} \Delta \varphi^{4}+\mathrm{a}_{5} \Delta \varphi^{5}+\mathrm{a}_{6} \Delta \varphi^{6} \\
& \mathrm{E}_{\mathrm{i}}=\mathrm{FE}+\mathrm{R} \operatorname{seno} \theta \\
& \mathrm{N}_{\mathrm{i}}=\mathrm{R}_{0}+\mathrm{FN}-\mathrm{R} \cos \theta
\end{aligned}
$$

Cuadro 2. Coeficientes para el cálculo de coordenadas planas en los sistemas LCRN y LCRS

\begin{tabular}{|l|c|c|}
\hline $\mathbf{a}_{\mathbf{i}}$ & Lambert Costa Rica Norte (LCRN) & Lambert Costa Rica Sur (LCRS) \\
\hline $\mathrm{a}_{1}$ & $-184332,51238 \cdot 10^{-2}$ & $-184316,54609 \cdot 10^{-2}$ \\
\hline $\mathrm{a}_{2}$ & $-9,72746 \cdot 10^{-4}$ & $-8,41218 \cdot 10^{-4}$ \\
\hline $\mathrm{a}_{3}$ & $-26,31931 \cdot 10^{-6}$ & $-26,32437 \cdot 10^{-6}$ \\
\hline $\mathrm{a}_{4}$ & $-0,03497 \cdot 10^{-8}$ & $-0,02998 \cdot 10^{-8}$ \\
\hline $\mathrm{a}_{5}$ & $-0,00564 \cdot 10^{-10}$ & $-0,00561 \cdot 10^{-10}$ \\
\hline $\mathrm{a}_{6}$ & $-0,00002 \cdot 10^{-12}$ & $-0,00002 \cdot 10^{-12}$ \\
\hline
\end{tabular}

Fuente: Elaboración propia a partir de IAGS, 1950

Las ecuaciones que permiten el cálculo de los coeficientes respetivos, así como el paso de coordenadas geodésicas a planas en proyección Lambert y demás elementos necesarios se pueden consultar, por ejemplo, en Grossmann (1976), Hooijberg (1998), Torge (2001), Leick (2004), Jekeli (2008) y (Hooijberg, 2008).

\section{Datum satelital CR90 y proyección CRTM90}

El proyecto de Catastro Multifinalitario, desarrollado en 1990 mediante el Acuerdo de Cooperación Técnico-Económica entre el Ministerio de Relaciones Exteriores de Costa Rica y el Reino de los Países Bajos, tenía dentro de uno de sus componentes el levantamiento de todos los predios del país (ILIS, 1988). Por otra parte, el rápido incremento en el uso del Sistema de Posicionamiento Global (GPS) constituyó la herramienta fundamental en el establecimiento de una nueva red geodésica nacional, 
diferente a la antigua red de triangulación tanto en configuración como en metodología de medición. Esta red fue medida usando receptores GPS de una frecuencia, cuyos resultados generaron el denominado datum Costa Rica 1990 (CR90). A pesar de que ya, para esa época, se contaba con una red global de estaciones permanentes, muchas de ellas usadas en la materialización del ITRF, el ajuste de la red se hizo asumiendo como coordenadas absolutas las de un antiguo punto ubicado en el edificio central del Catastro Nacional.

Como solución a la dualidad en la dirección norte-sur del sistema cartográfico Lambert, se propuso generar una nueva proyección cartográfica para el país, basada en el sistema UTM (adaptación de la proyección Gauss-Krüger). El sistema UTM divide el planeta en 60 fajas de $6^{\circ}$ de ancho, las cuales son numeradas desde el meridano de longitud $180^{\circ}$ de forma positiva hacia el este y con una reducción del meridiano central de cada faja en $-400 \mathrm{ppm}$. Dentro de esta numeración, Costa Rica se ubica entre las fajas número 16 y número 17. Para evitar esta nueva dualidad, pero en el sentido este-oeste, se hizo una adaptación de la UTM usando como meridiano central el de longitud $84^{\circ} \mathrm{O}$ para Costa Rica, denominándose como Costa Rica Transversa Mercator (CRTM). A esta proyección nacional se le denominó como CRTM90, generando cartografía a escala 1:25000.

\section{Datum satelital CR98 y proyección CRTM98}

Los resultados expuestos por Dörries y Roldán en (2004) tomaron como principales insumos las mediciones de la red GPS del año 1990 (definición del datum CR90) y mediciones GPS realizadas en los años 1991 y 1993, desarrolladas dentro del proyecto de investigación Costa Rica-Baja Sajonia (CoRBaS) (Niemeier et al, 1993). A diferencia del ajuste efectuado para la red CR90, en esta oportunidad se trabajó vinculando estas mediciones a puntos de medición continua pertenecientes al ITRF94. El ajuste de esta red usó el elipsoide WGS84, y sus coordenadas fueron determinadas a la época 1998,35. Estos resultados constituyen el denominado datum Costa Rica 1998 (CR98). Al igual que en el caso del CR90, para el datum CR98 se puede generar cartografía en proyección CRTM98, teniendo el cuidado de constatar que los datos estén vinculados al CR98. 


\section{Datum satelital CR05 y proyección CRTM05}

Dentro de las actividades del llamado Proyecto de Regularización de Catastro y Registro se encontraba la medición de una nueva red geodésica GPS, ajuste dentro del ITRF y propuesta cartográfica. Esta red sería adoptada como el nuevo datum nacional. El ajuste de esta red de 34 vértices se hizo contemplando estaciones internacionales pertenecientes al ITRF2000 para la época 2005,83. Como resultado de este trabajo, en el año 2007 el Decreto Ejecutivo 33937-MJ-MOPT establece en su artículo 1 que:

Se declara como datum horizontal oficial para Costa Rica, el CR05, enlazado al Marco Internacional de Referencia Terrestre (ITRF2000) del Servicio Internacional de Rotación de la Tierra (IERS) para la época de medición 2005,83, asociado al elipsoide del Sistema Geodésico Mundial (WGS84). Este datum está materializado a través de la denominada Red Geodésica Nacional de Referencia Horizontal CR05 de Primer Orden y su densificación al Segundo Orden, consistente en un conjunto vértices geodésicos situados sobre el terreno, dentro del ámbito del territorio nacional, establecidos físicamente mediante monumentos permanentes, sobre los cuales se han hecho medidas directas mediante el Sistema de Posicionamiento Global, estableciendo su interconexión y la determinación de su posición; y permitirá referenciar todos los levantamientos y actividades cartográficas y geodésicas que se efectúen en el Territorio Nacional.

Y en el artículo 2 se dice que:

Se declara como proyección oficial para la representación cartográfica, la Proyección Transversal de Mercator para Costa Rica con el acrónimo CRTM05... válida para todo el país.

Lo anterior implica que, todo producto que requiera la utilización de información geoespacial nacional debe estar referido al datum oficial CR05 y proyectada al plano cartográfico CRTM05 (Costa Rica, 2007).

Las ecuaciones que permiten el cálculo de los coeficientes respetivos, así como el paso de coordenadas geodésicas a planas en proyección UTM y demás elementos necesarios se pueden consultar, por ejemplo, en Grossmann (1976), Hooijberg (1998), Torge (2001), Leick (2004), Jekeli (2008) y (Hooijberg, 2008). 
La definición de las proyecciones CRTM son idénticas desde el punto de vista matemático, con excepción de la CRTM05, cuyo factor de escala cambia haciéndolo más cercano a uno. En el cuadro 2 se resumen los principales parámetros de los sistemas cartográficos CRTM, los cuales usan como superficie original de referencia el elipsoide WGS84, con un valor para el semieje a de $6378137 \mathrm{~m}$, para el semieje b de $6356752,314 \mathrm{~m}$ y para la primera excentricidad e $2=0,006694379908$. Se evidencia, como la única diferencia en la definición de la proyección es el factor de escala $\mathrm{k}_{0}$ del meridiano central.

Cuadro 3. Principales parámetros de los sistemas cartográficos Costa Rica Transversa Mercator CRTM

\begin{tabular}{|l|l|l|l|}
\hline \multicolumn{1}{|c|}{ Parámetro } & \multicolumn{1}{c|}{ CRTM90 } & \multicolumn{1}{c|}{ CRTM98 } & \multicolumn{1}{c|}{ CRTM05 } \\
\hline Elipsoide asociado & WGS84 & WGS84 & WGS84 \\
\hline Latitud origen & $\mathrm{j}_{0}=0^{\circ} 00^{\prime}$ & $\mathrm{j}_{0}=0^{\circ} 00^{\prime}$ & $\mathrm{j}_{0}=0^{\circ} 00^{\prime}$ \\
\hline Longitud origen & $\mathrm{l}_{0}=84^{\circ} 00^{\prime} \mathrm{O}$ & $\mathrm{l}_{0}=84^{\circ} 00^{\prime} \mathrm{O}$ & $\mathrm{l}_{0}=84^{\circ} 00^{\prime} \mathrm{O}$ \\
\hline $\begin{array}{l}\text { Factor de escala } \\
\text { meridiano central }\end{array}$ & $\mathrm{k}_{0}=0,999600$ & $\mathrm{k}_{0}=0,999600$ & $\mathrm{k}_{0}=0,999900$ \\
\hline Falso Norte & $\mathrm{FN}=0 \mathrm{~m}$ & $\mathrm{FN}=0 \mathrm{~m}$ & $\mathrm{FN}=0 \mathrm{~m}$ \\
\hline Falso Este & $\mathrm{FE}=500000,000 \mathrm{~m}$ & $\mathrm{FE}=500000,000 \mathrm{~m}$ & $\mathrm{FE}=500000,000 \mathrm{~m}$ \\
\hline
\end{tabular}

Fuente: Elaboración propia

El cálculo de las coordenadas cartográficas $(\mathrm{N}, \mathrm{E})$ a partir de las coordenadas geodésicas $(\varphi, \lambda)$ se hace considerando el conjunto de ecuaciones (2). Donde $\mathrm{D} \varphi$ es la diferencia de latitud entre el punto nuevo y la latitud $10^{\circ} \mathrm{N}$. El valor $\mathrm{D} \lambda$ es la diferencia entre la longitud del punto nuevo y la longitud $84^{\circ} \mathrm{O}$, considerando el signo de las coordenadas. El parámetro $\mathrm{G}$ es la longitud del arco de meridiano desde el Ecuador a la latitud $10^{\circ}$ norte y tiene un valor de $1105854,833 \mathrm{~m}$ para el elipsoide WGS84. El parámetro $\mathrm{k}_{0}$ es el factor de escala del meridiano central (ver cuadro 3). Los valores de los coeficientes $\mathrm{a}_{\mathrm{ij}}$ se presentan en el cuadro 4. 


$$
\begin{aligned}
& \Delta \mathrm{N}^{\prime}=a_{10} \Delta \varphi+a_{20} \Delta \varphi^{2}+a_{02} \Delta \lambda^{2}+a_{30} \Delta \varphi^{3}+a_{12} \Delta \varphi \Delta \lambda^{2}+a_{40} \Delta \varphi^{4}+ \\
& a_{22} \Delta \varphi^{2} \Delta \lambda^{2}+a_{04} \Delta \lambda^{4}+a_{50} \Delta \varphi^{5}+a_{32} \Delta \varphi^{3} \Delta \lambda^{2}+a_{14} \Delta \varphi \Delta \lambda^{4}+\ldots \\
& =a_{01} \Delta \lambda+a_{11} \Delta \varphi \Delta \lambda+a_{21} \Delta \varphi^{2} \Delta \lambda+a_{03} \Delta \lambda^{3}+a_{31} \Delta \varphi^{3} \Delta \lambda+ \\
& a_{13} \Delta \varphi \Delta \lambda^{3}+a_{41} \Delta \varphi^{4} \Delta \lambda+a_{23} \Delta \varphi^{2} \Delta \lambda^{3}+a_{05} \Delta \lambda^{5}+\ldots
\end{aligned}
$$

Cuadro 4. Coeficientes para el cálculo de coordenadas cartográficas en las proyecciones CRTM

\begin{tabular}{|c|r|c|r|}
\hline Coeficiente & \multicolumn{1}{|c|}{ Valor } & Coeficiente & Valor \\
\hline $\mathrm{a}_{10}$ & 6337358,1100 & $\mathrm{a}$ & 1103101,2998 \\
\hline $\mathrm{a}_{20}$ & 10883,9934 & $\mathrm{a}^{14}$ & 6281872,8296 \\
\hline $\mathrm{a}_{02}$ & 545417,8846 & $\mathrm{a}^{01}$ & $-1100470,6870$ \\
\hline $\mathrm{a}_{30}$ & 19956,7312 & $\mathrm{a}^{11}$ & $-3122429,6801$ \\
\hline $\mathrm{a}_{12}$ & 2997671,1627 & $\mathrm{a}^{21}$ & 990475,2476 \\
\hline $\mathrm{a}_{40}$ & $-3582,8519$ & $\mathrm{a}^{33}$ & 172800,8246 \\
\hline $\mathrm{a}_{22}$ & $-1085687,4633$ & $\mathrm{a}^{31}$ & $-894370,1807$ \\
\hline $\mathrm{a}_{04}$ & 221634,9321 & $\mathrm{a}^{13}$ & 225949,7249 \\
\hline $\mathrm{a}_{50}$ & $-7723,2841$ & $\mathrm{a}^{41}$ & $-2305563,8829$ \\
\hline $\mathrm{a}_{32}$ & $-1949714,4689$ & $\mathrm{a}^{23}$ & 218688,6700 \\
\hline
\end{tabular}

Fuente: Elaboración propia

\section{Comparación de los diferentes datum de Costa Rica}

Uno de los principales resultados generados por Dörries y Roldán en (2004) fue precisamente detectar y cuantificar las diferencias obtenidas entre el datum convencional de Ocotepeque y el datum satelital CR98, con valor promedio para el país de $265 \mathrm{~m}$. También, se cuantificó la diferencia entre el datum CR90 y el datum CR98, ambos definidos a partir de mediciones GPS. Este valor promedio para el país es de 7,5 m, el cual se debe, fundamentalmente a la falta del vínculo del datum CR90 a estaciones 
pertenecientes al ITRF. En el cuadro 5 se comparan las principales características de los cuatro datum expuestos anteriormente.

Cuadro 5. Resumen de los diferentes datum y proyecciones cartográficas asociadas de Costa Rica

\begin{tabular}{|l|l|l|l|l|}
\hline \multicolumn{1}{|c|}{ Datum } & \multicolumn{1}{c|}{$\begin{array}{c}\text { Tipo de } \\
\text { mediciones }\end{array}$} & \multicolumn{1}{c|}{$\begin{array}{c}\text { Vínculo al } \\
\text { ITRF }\end{array}$} & \multicolumn{1}{|c|}{ Elipsoide } & \multicolumn{1}{|c|}{ Proyección } \\
\hline Ocotepeque & Convencionales & NO & Clarke 1866 & LCRN y LCRS \\
\hline CR90 & Satelitales GPS & NO & WGS84 & CRTM90 \\
\hline CR98 & Satelitales GPS & $\begin{array}{l}\text { ITRF1994, } \\
\text { época 1998,35 }\end{array}$ & WGS84 & CRTM98 \\
\hline CR05 & Satelitales GPS & $\begin{array}{l}\text { ITRF2000, } \\
\text { época 2005,83 }\end{array}$ & WGS84 & CRTM05 \\
\hline
\end{tabular}

Fuente: Elaboración propia

\section{¿Con qué se cuenta y a dónde se puede ir?}

Los criterios que definen una proyección cartográfica se derivan, fundamentalmente, de las características que se le quiera dar a la carta resultante, por ejemplo, equidistancia, equivalencia o conformidad como es el caso de las proyecciones geodésicas. El resto de parámetros necesarios dependen del elipsoide escogido como superficie de referencia y de los propios parámetros de las funciones de proyección. Es decir, no interesa ni la ubicación ni la orientación del elipsoide escogido o el datum de partida, ya que la finalidad de las proyecciones cartográficas es precisamente proyectar la superficie de referencia en el plano (Moya y Cedeño, 2017). Sin embargo, del datum si dependen las coordenadas geodésicas, las cuales condicionan las coordenadas cartográficas. De esta manera, se puede tener la misma proyección cartográfica, pero si las coordenadas geodésicas de partida provienen de datum diferentes, sus valores serán diferentes en el plano, por ejemplo, un punto que tenga coordenadas geodésicas asociadas al datum CR90 y al datum CR98.

El conocimiento para transformar coordenadas geodésicas de un datum a otro o coordenadas entre planos cartográficos es fundamental, sobre todo en Costa Rica, donde se tienen muchas variantes. La existencia de al menos cuatro datum nacionales con sus respectivas proyecciones cartográficas conducen a la necesidad de conocer exactamente el origen de la información, de manera que se pueda hacer una transformación correcta. 
En la siguiente figura se muestra un esquema que presenta qué tipo de transformación se debe realizar dependiendo de los datos que se tengan y hacia donde se quiera ir.

Figura 1. Esquema de transformación entre el datum convencional Ocotepeque y los datum satelitales CR90, CR98 y CR05

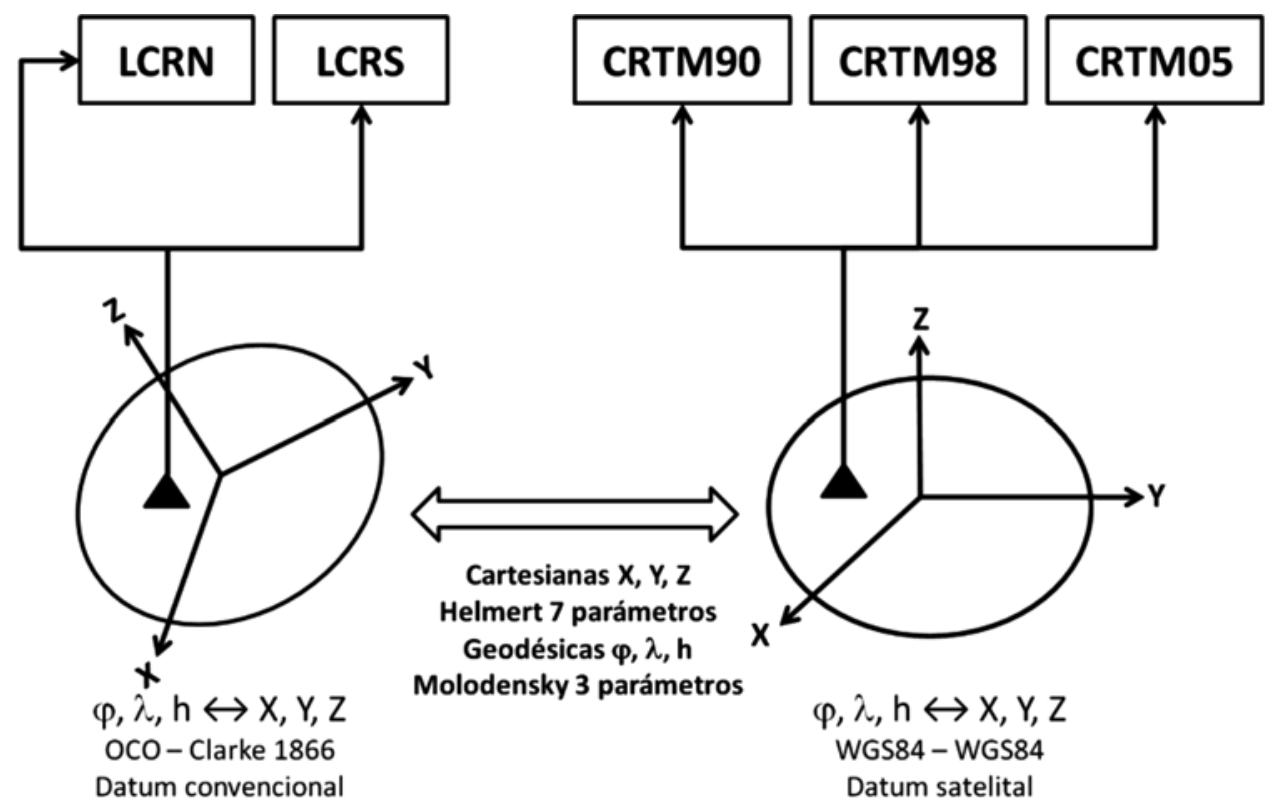

Fuente: Elaboración propia

Por ejemplo, se pueden presentar los siguientes casos desde el punto de vista horizontal:

- Contar con información de coordenadas geodésicas $(\varphi, \lambda)$ sobre el elipsoide Clarke 1866 vinculado al datum Ocotepeque. Estas coordenadas deben proyectarse a los planos cartográficos LCRN o LCRS respectivamente, dependiendo de la zona de ubicación en el terreno, sin embargo, si se quisiera también se pueden calcular coordenadas planas tanto en LCRN como en LCRS para cualquier punto. 
- Contar con información en coordenadas geodésicas $(\varphi, \lambda)$ sobre el elipsoide WGS84 vinculado con el datum CR90, éstas deben proyectarse al plano cartográfico CRTM90.

- Contar con información en coordenadas geodésicas $(\varphi, \lambda)$ sobre el elipsoide WGS84 vinculado con el datum CR98, éstas deben proyectarse al plano cartográfico CRTM98.

- Contar con información en coordenadas geodésicas $(\varphi, \lambda)$ sobre el elipsoide WGS84 vinculado con el datum CR05, éstas deben proyectarse al plano cartográfico CRTM05.

En el caso que se cuente con la terna de coordenadas geodésicas $(\varphi$, $\lambda, h)$ en un sistema, y se desee conocer su ubicación en otros sistemas diferentes, debería aplicarse una transformación de datum (Moya y Cedeño, 2017). El cálculo de estos parámetros se hace con base en las correspondientes coordenadas geocéntricas $(\mathrm{X}, \mathrm{Y}, \mathrm{Z})$. En caso clásico es realizar una transformación semejante de Helmert en sus diferentes variantes, siendo la clásica de 7 parámetros la más aplicable en el caso de tener que relacionar un datum convencional con un datum satelital. Estos 7 parámetros representan tres traslaciones (DX, DY, DZ) entre los geocentros de ambos sistemas, tres rotaciones (RX, RY, RZ) alrededor de cada uno de los ejes $\mathrm{y}$ un factor de escala $\mathrm{m}$. La formulación respectiva se puede consultar con mayor detalle en (Hofmann-Wellenhof et al, 2008)

Es importante dejar claro que en Costa Rica solamente se han determinado parámetros de transformación entre el datum Ocotepeque (elipsoide Clarke) y el datum CR98 (elipsoide WGS84), época 1998.35, cuyos valores se muestran en el cuadro 6. Estos valores son el resultado al aplicar la variante conocida como Molodensky-Badekas (Dörries y Roldán, 2004). 
Cuadro 6. Parámetros de transformación entre los datum CR98 y datum Ocotepeque

\begin{tabular}{|l|l|l|l|l|}
\hline \multicolumn{1}{|c|}{ Parámetro } & \multicolumn{1}{|c|}{ Valor } & \multicolumn{1}{c|}{ Unidades } & \multicolumn{1}{c|}{ Errores } & \multicolumn{1}{c|}{ Unidades } \\
\hline $\begin{array}{l}\text { Coordenada } \mathrm{X} \\
\text { del baricentro }\end{array}$ & 617749,712 & $\mathrm{~m}$ & & \\
\hline $\begin{array}{l}\text { Coordenada } \mathrm{Y} \\
\text { del baricentro }\end{array}$ & $-6250547,734$ & $\mathrm{~m}$ & & \\
\hline $\begin{array}{l}\text { Coordenada } \mathrm{Z} \\
\text { del baricentro }\end{array}$ & 1102063,610 & $\mathrm{~m}$ & & \\
\hline Traslación en $\mathrm{X}$ & $-213,116$ & $\mathrm{~m}$ & 0,428 & $\mathrm{~m}$ \\
\hline Traslación en $\mathrm{Y}$ & $-9,358$ & $\mathrm{~m}$ & 0,428 & $\mathrm{~m}$ \\
\hline Traslación en $\mathrm{Z}$ & 74,946 & $\mathrm{~m}$ & 0,428 & $\mathrm{~m}$ \\
\hline Rotación en $\mathrm{X}$ & $-1,14 \mathrm{E}-06$ & radianes & $7,8 \mathrm{E}-06$ & radianes \\
\hline Rotación en $\mathrm{Y}$ & $2,98 \mathrm{E}-07$ & radianes & $3,4 \mathrm{E}-06$ & radianes \\
\hline Rotación en $\mathrm{Z}$ & $-3,10 \mathrm{E}-08$ & radianes & $5,4 \mathrm{E}-06$ & radianes \\
\hline Factor de escala & 5,22 & ppm & 3,4 & ppm \\
\hline
\end{tabular}

Fuente: (Dörries y Roldán, 2004)

Esto quiere decir, que para poder tener información dentro del datum oficial de Costa Rica CR05 que no se haya determinado exactamente dentro de este datum, deben hacerse procesos intermedios de conversión y transformación de coordenadas, dependiendo de la información de partida. Considerar siempre que cada datum nacional tiene asociado su elipsoide y su proyección cartográfica (ver cuadro 5). De esta manera, se pueden presentar las siguientes posibilidades en las que por supuesto, se puede realizar el procedimiento inverso:

A. Coordenadas geodésicas $(\varphi, \lambda)$ en el datum Ocotepeque, sin altura elipsoídica (h). Es el caso más común:

1. Calcular las correspondientes coordenadas cartográficas $(\mathrm{N}$, E) en el plano LCRN.

2. Calcular coordenadas cartográficas $(\mathrm{N}, \mathrm{E})$ en el plano CRTM98, usando una transformación polinómica de $4^{\circ}$ grado (Dörries y Roldán, 2004).

3. Calcular las correspondientes coordenadas cartográficas $(\mathrm{N}$, E) en el plano CRTM05, usando una transformación bidimensional de Helmert. 
B. Coordenadas geodésicas $(\varphi, \lambda)$ en el datum Ocotepeque, con altura elipsoídica (h). Es el caso ideal, sin embargo, no es frecuente el conocimiento de la altura elipsoídica relativa al datum Ocotepeque, elipsoide Clarke 1866:

1. Calcular las correspondientes coordenadas geocéntricas (X, Y, Z) sobre el elipsoide Clarke 1866, datum Ocotepeque.

2. Aplicar una transformación espacial de Helmert para calcular las coordenadas geocéntricas (X, Y, Z) en el datum CR98, elipsoide WGS84 (usar parámetros del cuadro 6).

3. Calcular las correspondientes coordenadas geodésicas $(\phi, \lambda, \mathrm{h})$ sobre el elipsoide WGS84, datum CR98.

4. Calcular las coordenadas cartográficas $(\mathrm{N}, \mathrm{E})$ en el plano CRTM98.

5. Calcular las correspondientes coordenadas cartográficas $(\mathrm{N}$, E) en el plano CRTM05, usando una transformación bidimensional de Helmert.

C. Coordenadas geodésicas $(\varphi, \lambda, \mathbf{h})$ en el datum CR90. Es el caso en que se tienen datos derivados de mediciones GPS. Aunque se conozca la altura elipsoídica, la transformación se debe hacer entre planos cartográficos:

1. Calcular las correspondientes coordenadas cartográficas $(\mathrm{N}$,

E) en el plano CRTM90. Se pueden hacer dos variantes:

1.1. Calcular las coordenadas cartográficas $(\mathrm{N}, \mathrm{E})$ en el plano CRTM98, usando una transformación bidimensional del Helmert.

1.2. Calcular las coordenadas cartográficas $(\mathrm{N}, \mathrm{E})$ en el plano CRTM05, usando una transformación bidimensional del Helmert.

2. Otra posibilidad es pasar las coordenadas cartográficas $(\mathrm{N}$, E) a coordenadas cartográficas $(\mathrm{N}, \mathrm{E})$ sobre el plano LCRN o LCRS, según la ubicación de punto usando una transformación polinómica de $3^{\circ}$ grado. Luego pasar al plano CRTM98 con una transformación polinómica $4^{\circ}$ grado y luego al plano CRTM05 con una transformación bidimensional de Helmert. 


\section{Un ejemplo numérico}

El vértice geodésico BUVIS ubicado en el Cerro de La Muerte, es uno de los pocos puntos en Costa Rica que ha sido medido directamente en todos los proyectos que se han generado datum nacional. Partiendo de las coordenadas geodésicas de este punto, se han calculado sus correspondientes coordenadas cartográficas y luego por los diferentes procesos de transformación descritos (ver figura 1), se calcularon las coordenadas respectivas y las diferencias.

En el cuadro 7 se presentan los resultados donde la nomenclatura usada es: $\mathrm{P} 3$ para transformación polinómica de $3^{\circ}$ grado, $\mathrm{P} 4$ para transformación polinómica de $4^{\circ}$ grado, $\mathrm{H} 7$ para transformación de Helmert de 7 parámetros con los valores del cuadro 6 y H4 para transformación Helmert de 4 parámetros. Los cálculos respectivos se hicieron usando los archivos en formato *.xls generados en el marco del proyecto CR05 y que fueron entregados y puestos a disposición de los usuarios. 
Jorge Moya Zamora, Bepsy Cedeño Montoya. Different datum and cartographic projections from Costa Rica: generalities and relations

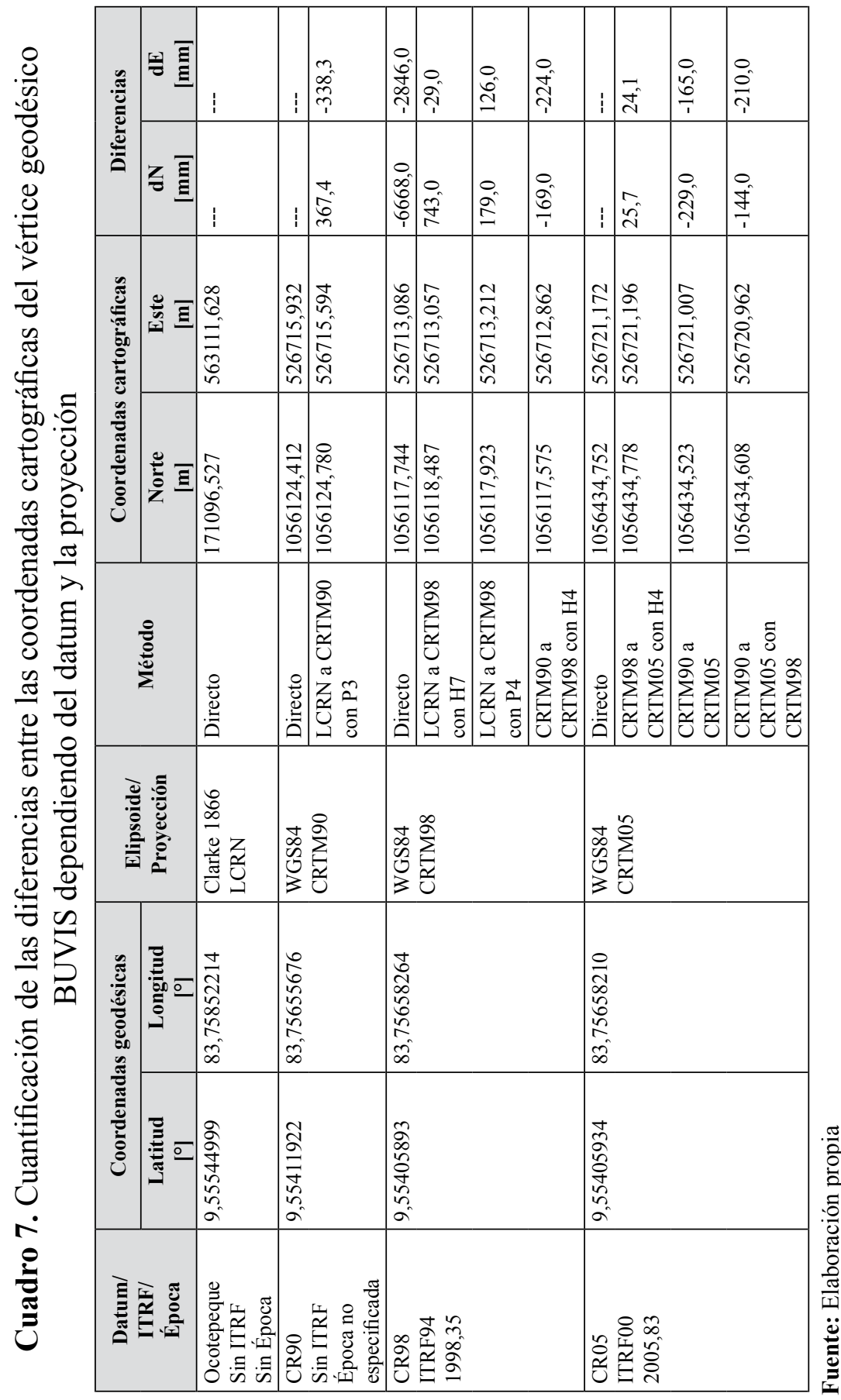




\section{Las coordenadas determinadas por SIRGAS}

La red de estaciones del Sistema Geocéntrico para las Américas (SIRGAS) tiene una distribución continental que supera las 400 estaciones. En Costa Rica se cuenta con un total de 14 estaciones integradas oficialmente a la red SIRGAS-CON, sin embargo, actualmente están en operación solamente 12. Esta red nacional de estaciones constituye hoy día la referencia geodésica más exacta del país, ya que sus coordenadas geocéntricas se determinan semanalmente con altos estándares internacionales y dentro de un marco de referencia dinámico. A pesar de alta exactitud en las coordenadas de esta red, no sería correcto calcular sus coordenadas cartográficas en la proyección oficial CRTM05, simplemente por el hecho de que no están en el mismo datum.

Como parte de las actividades de este proyecto se determinaron por medio de ajuste geodésico los 6 parámetros de transformación entre el datum CR05 y el datum IGb08 (marco SIRGAS). Para el cálculo de este conjunto de parámetros se usaron inicialmente como puntos idénticos las estaciones ETCG, LIBE, LIMN, NEIL, NICY, PUNT, RIDC y SAGE. Estas estaciones poseen coordenadas referidas al datum CR05, época 2005,83. Adicionalmente, se usaron como coordenadas SIRGAS las de la solución final de la semana 1803, época 2014,58. La primera determinación de parámetros brindó residuales para la estación NICY del orden de los $-118,0 \mathrm{~mm}$ en $\mathrm{X}$, de $-86,0 \mathrm{~mm}$ en $\mathrm{Y}$ y de $-178,0 \mathrm{~mm}$ en $\mathrm{Z}$. Una segunda determinación de parámetros excluyendo a NICY, brindó residuales para la estación LIBE del orden $-111,0 \mathrm{~mm}$ en la coordenada Z. Se realizó una tercera determinación excluyendo tanto a NICY como a LIBE cuyos resultados se presentan en el cuadro 8. 
Cuadro 8. Parámetros de transformación del datum SIRGAS, época 2014,58 (semana 1803) al datum CR05, época 2005,83

\begin{tabular}{|l|r|c|c|c|}
\hline \multicolumn{1}{|c|}{ Parámetro } & \multicolumn{1}{c|}{ Valor } & Unidades & Error & Unidades \\
\hline Coordenada X del baricentro & 681700,5963 & $\mathrm{~m}$ & & \\
\hline Coordenada Y del baricentro & $-6251273,9638$ & $\mathrm{~m}$ & & \\
\hline Coordenada Z del baricentro & 1062332,0346 & $\mathrm{~m}$ & & \\
\hline Traslación en X & $-0,1092$ & $\mathrm{~m}$ & 0,0162 & $\mathrm{~m}$ \\
\hline Traslación en Y & $-0,1620$ & $\mathrm{~m}$ & 0,0162 & $\mathrm{~m}$ \\
\hline Traslación en Z & $-0,1758$ & $\mathrm{~m}$ & 0,0162 & $\mathrm{~m}$ \\
\hline Rotación en X & $-0,1689$ & “ & 0,0740 & “ \\
\hline Rotación en Y & 0,1528 & “ & 0,0369 & “ \\
\hline Rotación en Z & $-0,0052$ & “ & 0,0567 & “ \\
\hline
\end{tabular}

Fuente: Elaboración propia

\section{Conclusiones}

- $\quad$ En Costa Rica los diferentes datum y sus proyecciones cartográficas asociadas son la base de innumerable información geoespacial. Por consiguiente, se cuenta con datos que están referidos al datum Ocotepeque, elipsoide Clarke 1866, al datum CR90, elipsoide WGS84, al datum CR98, elipsoide WGS84 y en este momento al datum oficial CR05, elipsoide WGS84. Toda esta información debe ser tratada adecuadamente en aras de poder extraer el mayor provecho dependiendo de los objetivos de los usuarios.

- Las relaciones entre los diferentes datum nacionales es conocida, por lo que efectuar los procesos de conversión y transformación de coordenadas, dependiendo de la información de partida y hacia donde se necesita llevar, es fundamental en aras de poder alimentar adecuadamente, por ejemplo, Sistemas de Información Geográfica (SIG) con datos referidos correctamente y por consiguiente obtener resultados de calidad, pero sobretodo correctamente georreferenciados.

- $\quad$ El usuario debe tener presente que en Costa Rica se han desarrollado, fundamentalmente dos procesos de transformación de coordenadas para poder referir información al actual datum oficial del país. Los 7 parámetros determinados por Dörries y Roldán (2004) permiten transformar información del datum convencional de Ocotepeque al datum CR98 y viceversa. Sin embargo, como ya fue expuesto, el 
proceso estricto de transformación implica el conocimiento de la altura elipsoídica en datum Ocotepeque, de manera de poder convertir posteriormente a coordenadas geocéntricas, y de éstas, a coordenadas geocéntricas referidas al datum CR98 para finalmente calcular coordenadas, las cartográficas. A falta de esta coordenada vertical, en la mayoría de los casos se deben aplicar transformaciones polinómicas, las cuales no son semejantes, son afines, pero es lo que se tiene. Básicamente si se parte de coordenadas cartográficas referidas a los planos Lambert, éstas se pueden llevar al plano CRTM05 aplicando diferentes variantes.

- El caso más común que se presenta actualmente en Costa Rica implica trasladar información geoespacial, que fue levantada, procesada y referida al datum Ocotepeque, cartografía en LCRN y LCRS, al datum CR05, proyección CRTM05, según lo exige la legislación nacional. Este proceso debe efectuarse con suma cautela, ya que el camino estricto implicaría como paso inicial aplicar parámetros de transformación entre este datum y el datum CR05. Estos parámetros no existen. La forma adecuada de poder hacer la referencia, por ejemplo, de información digitalizada de las hojas del mapa básico a la proyección CRTM05 implica un proceso de transformación de coordenadas planas como el descrito anteriormente.

- Es claro que, aunque los sistemas informáticos, como por ejemplo los GIS u otros programas de proyecciones cartográficas tienen integrados los algoritmos correctos de conversión y transformación de coordenadas, es responsabilidad del usuario alimentarlos con la información correcta desde el punto de vista del vínculo geodésico. Lo anterior está relacionado con el hecho de que, aunque matemáticamente las funciones de proyección permitan, por ejemplo, calcular coordenadas planas en la proyección LCRN partiendo de coordenadas geodésicas vinculadas al datum CR90, CR98 o CR05 o, por el contrario, calcular coordenadas geodésicas partiendo de coordenadas planas en CRTM05, datum CR05, y asumir que las coordenadas resultantes están vinculadas al datum Ocotepeque, no son procedimientos correctos. Debe ponerse especial atención en cuáles caminos pueden realizarse, dependiendo siempre de la información inicial y hacia donde se quiera o pueda llegar. 
- $\quad$ El caso numérico presentado y cuyos resultados se resumen en el cuadro 7, evidencia la influencia del datum en las coordenadas geodésicas y por consiguiente en las coordenadas cartográficas. Por ejemplo, la diferencia en las coordenadas geodésicas referidas al datum Ocotepeque y al datum CR98 es de aproximadamente 155 $\mathrm{m}$ en la latitud y de $216 \mathrm{~m}$ en longitud con una resultante de $266 \mathrm{~m}$ totalmente concordante con Dörries y Roldán (2004). Además, si se comparan las coordenadas geodésicas entre los datum CR98 y CR05 se tienen diferencias en latitud y longitud de unos 1,5 y 1,9 milésimos de segundo de arco, que representan aproximadamente 5 $\mathrm{cm}$ entre ambas determinaciones.

- Los diferentes procesos de conversión y transformación de coordenadas, cuyos parámetros fueron derivados de los datos originales, muestran por ejemplo que en la aplicación de la transformación polinómica P3 se obtuvieron diferencias promedio del orden de los 35 $\mathrm{cm}$ para el datum CR90.

- $\quad$ En el caso de la transformación espacial de Helmert H7 se partió de coordenadas cartográficas oficiales LCRN y con el valor modelado de la altura elipsoídica, según Dörries y Roldán (1999) se obtuvieron las respectivas coordenadas geocéntricas en Clarke 1866, se transformaron a geocéntricas en WGS84, luego se convirtieron a coordenadas geodésicas y finalmente se proyectaron al plano CRTM98. Las discrepancias obtenidas fueron de $74,3 \mathrm{~cm}$ en la coordenada norte y $2,9 \mathrm{~cm}$ en la coordenada este. Por su parte, la aplicación de la transformación polinómica P4 da diferencias promedio de 148 $\mathrm{mm}$, mientas que usando una transformación bidimensional $\mathrm{H} 4$ las diferencias son en promedio de $200 \mathrm{~mm}$.

- $\quad$ Para el datum CR05, las diferencias promedio de $25 \mathrm{~mm}$ se obtuvieron al aplicar la transformación bidimensional $\mathrm{H} 4$, mientras que las transformaciones de CRTM90 a CRTM05 directa ofrece diferencias promedio de $197 \mathrm{~mm}$, y de CRTM90 a CRTM05 con CRTM98 intermedio ofrece discrepancias de $177 \mathrm{~mm}$.

- Las proyecciones CRTM90 y CRTM98 son iguales en su determinación, diferenciándose claro está en el datum. Por su parte, la proyección CRTM05 usa un factor de escala de -100 ppm. Partiendo de las coordenadas del punto BUVIS en el datum CR90, si se comparan 
con las coordenadas obtenidas en el datum CR98, se tiene como resultante 7,2 m concordante con lo reportado por Dörries y Roldán (2004) producto del vínculo del datum CR98 al ITRF1994.

- Se evidencia a partir de las comparaciones de coordenadas presentadas en el cuadro 7, que existen discrepancias, las cuales son producto de considerar diferentes datum como origen de la información. Sin embargo, los valores presentados en el ejemplo deben interpretarse como una muestra de las diferencias que eventualmente puedan obtenerse en diferentes aplicaciones. Estas pueden aumentar si, además, el usuario no es consciente del vínculo geodésico de los datos de entrada, y aún más de la configuración de las herramientas usadas en los distintos procesos de transformación de la información geoespacial.

- Las diferencias obtenidas por un incorrecto tratamiento de la información en cuanto a su origen, producirá discrepancias que pueden ser desde algunos milímetros hasta algunos metros. Estas diferencias muy probablemente no sean "significativas" desde el punto de vista de los objetivos (por ejemplo, la escala de los mapas resultantes), pero no deja de ser indispensable su conocimiento, consideración y aplicación.

- $\quad$ El conjunto de parámetros determinados entre el datum CR05 y el datum definido por SIRGAS, debe considerarse como una primera aproximación. En el cálculo de estos parámetros, lamentablemente se tuvo que excluir los puntos NICY y LIBE por altos residuales. Se debe notar que estos dos puntos se encuentran en el sector occidental de Costa Rica y, considerando las épocas en las que se hizo la determinación de los parámetros, estas dos estaciones estuvieron afectadas por el terremoto de Nicoya de 2012. Lo anterior implica, que el conjunto de parámetros se calculó sin contemplar información en el oeste del país. Se recomienda a los usuarios tener cautela en el uso de estos valores y considerar lo expuesto anteriormente. 
Jorge Moya Zamora, Bepsy Cedeño Montoya. Different datum and cartographic projections from Costa Rica: generalities and relations

\section{Referencias}

Costa Rica. (2007). Decreto ejecutivo 33797-MJ-MOPT. Ministerio de Justica y Gracia. Costa Rica.

Dörries, E. y J. Roldán (1999). Estudio Comparativo del Datum Geodesico de Ocotepeque y el Datum Satelitario del Sistema WGS84. Informe Final Proyecto de Investigación. Escuela de Topografía, Catastro y Geodesía. Universidad Nacional.

Dörries, E. y J. Roldán (2004). El Datum Geodésico de Ocotepeque y el Datum Satelitario del Sistema WGS84. Revista UNICIENCIA, 21(1 y 2), 117-125.

Grosmann, W. (1976). Geodätische Rechnungen und Abbildungen in der Landesvermessung. Verlag Konrad Wittwer, Stuttgart.

Grafarend, E., R. You y R. Syffus (2014) Map Projections Cartographic Information Systemns. Second Edition. Springer-Verlag Berlin Heidelberg. DOI 10.1007/978-3-642-36494-5

Hooijberg, M. (1998). Practical Geodesy Using Computer. Springer-Verlag Berlin, Alemania.

Hooijberg, M. (2008). Geometrical Geodesy Using Information and Computer Technology. Springer-Verlag Berlin. Alemania

Hoffmann-Weelenhof, B., H. Lichtenegger y E. Wasle (2008). GNSS Global Navigation Satellite Systems GPS, GLONASS, Galileo \& more. Springer Wein, New York. Estados Unidos.

IAGS. (1950). Proyección Lambert Para Costa Rica. InterAmerican Geodetic Suvey, Estados Unidos de América.

ILIS (1988). Catastro Multifinalitario Programa de Mejoramiento del Catastro Nacional. Reino de los Países Bajos. Dirección General para la Cooperación Internacional (DGIS).

Jekeli, C. (2006). Geometric Reference System in Geodesy. Division of Geodesy and Geospatial Science. School of Earth Sciences. Ohio State University.

Leick, A. (2004). GPS Satellite Surveying. Editorial Wiley. Tercera Edición. Estados Unidos. 464 pp. ISBN: 978-0-471-05930-1

Moya, J. y B. Cedeño (2017). Conceptos básicos en geodesia como insumo para un tratamiento adecuado de la información geoespacial. Revista Geográfica de América Central, 58(1). ISSN 1011-484X. 71-100.Niemeier, W., J. Roldán, L. Aguilar, H. Pelzer, A. Bagge, 
W. Augath y W. Seifert (1993). The Project CORBAS - determination of recent crustal movements in Costa Rica. Recent Geodetic and Gravimetric Research in Latin America. IAG Symposium No. 111. Vienna Austria

Torge, W. (2001). Geodesy. 3rd. Edition. Walter de Gruyter-Berlin-New York. $416 \mathrm{pp}$.

\section{Lista de siglas y acrónimos}

\begin{tabular}{|l|l|}
\hline LCRN & Proyección Lambert Costa Rica Norte \\
\hline LCRS & Proyección Lambert Costa Rica Sur \\
\hline CR90 & Datum Costa Rica 1990 \\
\hline CRTM90 & Proyección Costa Rica Transversa Mercator 1990 \\
\hline CR98 & Datum Costa Rica 1998 \\
\hline CRTM98 & Proyección Costa Rica Transversa Mercator 1998 \\
\hline CR05 & Datum Costa Rica 2005 \\
\hline CRTM05 & Proyección Costa Rica Transversa Mercator 2005 \\
\hline UTM & Universal Transversa Mercator \\
\hline H7 & Transformación tridimensional semejante de Helmert de 7 parámetros \\
\hline H4 & Transformación bidimensional semejante de Helmert de 4 parámetros \\
\hline P3 & Transformación polinómica de 3 grado \\
\hline P4 & Transformación polinómica de 4 grado \\
\hline ITRF & $\begin{array}{l}\text { International Terrestrial Reference Frame (Marco Internacional } \\
\text { Terrretre de Referencia) }\end{array}$ \\
\hline$(X, Y, Z)$ & Coordenadas geocéntricas \\
\hline$(\varphi, \lambda, h)$ & Coordenadas geodésicas \\
\hline WGS84 & Elipsoide World Geodetic System 1984 \\
\hline SIRGAS & Sistema de Referencia Geocéntrica para las Américas \\
\hline DX, DY, DZ & Traslaciones en X, Y, Z entre los geocéntros de los sistemas \\
\hline RX, RY, RZ & Rotaciones entre los ejes X, Y, Z de los sistemas \\
\hline$m$ & Factor de escala \\
\hline &
\end{tabular}

